\title{
Guest editorial: Advances in RFID technology
}

\author{
Quan Z. Sheng · Sherali Zeadally • Zongwei Luo • \\ Jen-Yao Chung $\cdot$ Zakaria Maamar
}

Published online: 24 July 2009

(C) Springer Science + Business Media, LLC 2009

This special issue aims at presenting the latest developments, trends, and research solutions for RFID technology, which received an enthusiastic response. There were 40 submissions, including six extended from the best papers of IWRT2008 (the 2nd International Workshop on RFID Technology, Barcelona, Spain), AiR2008 (the First International Workshop on Advances in RFID, Xi'an, China), and Industrial Track of SUTC2008 (IEEE International Conference on Sensor Networks, Ubiquitous, and Trustworthy Computing, Taichung, Taiwan). Eleven papers were selected after several rounds of review by the guest editors and the invited reviewers. These papers cover a wide range of

\section{Q. Z. Sheng $(\bowtie)$}

School of Computer Science,

The University of Adelaide, Adelaide,

South Australia 5005, Australia

e-mail: qsheng@cs.adelaide.edu.au

\section{S. Zeadally}

Department of Computer Science and Information Technology, University of the District of Columbia, Washington, DC 20008, USA

\section{Z. W. Luo}

E-business Technology Institute,

The University of Hong Kong,

Hong Kong, China

\section{J.-Y. Chung}

Industry Technology and Solutions,

IBM T. J. Watson Research Center,

New York, NY, USA

\section{Z. Maamar}

College of Information Technology,

Zayed University, Dubai,

United Arab Emirates topics in RFID research that reflect some key directions in this active yet diverse research area.

The first short paper, by the Guest Editors of this special issue Sheng et al. (2009), outlines some of the challenges and key research directions in the RFID area. Three articles in this special issue deal with security and privacy of RFID technology. The paper by Mitrokotsa et al., "Classifying RFID Attacks and Defenses" Mitrokotsa et al. (2009), provides a comprehensive overview of possible attacks that RFID networks may face. The authors categorize the weaknesses of the existing RFID communications, which could serve a foundation for better understanding of RFID attacks and defense against these attacks. The paper by Zuo, "Secure and Private Search Protocol for RFID Systems" Zuo (2009), proposes a set of protocols that enable an RFID reader to search for particular tags based on their identities or certain criteria. The third paper by Zhou et al., "A Lightweight Anti-Desynchronization RFID Authentication Protocol" Zhou et al. (2009), proposes a lightweight antidesynchronization RFID authentication protocol that prevents attackers from de-synchronizing the shared secret between an RFID tag and its back-end server.

The next three articles focus on integration frameworks for RFID application development. The paper by Schwieren and Vossen, "ID-Services: An RFID Middleware Architecture for Mobile Applications" Schwieren and Vossen (2009), presents ID-Services, an RFID middleware architecture to support mobile applications. The paper by Ziekow and Günther, "Sharing RFID and Complex Event Data Among Organizations" Ziekow and Günther (2009), concerns the performance issue of the current standardization efforts (e.g., EPCglobal Network) in sharing and integrating 
RFID event data in distributed environments. The authors propose a peer-to-peer (P2P) based architecture for event-based interaction and a new method for in-network query processing. The paper by Sengupta and Schiller, "FlexRFID: A Design, Development and Deployment Framework for RFID-based Business Applications" Sengupta and Schiller (2009), describes FlexRFID, a modeling template for rapid prototyping RFID applications. It also describes a real-life RFIDenabled car parking system developed by FlexRFID.

The paper by Meng et al., "Automated Management of Assets Based on RFID Triggered Alarm Messages" Meng et al. (2009), reports the implementation of an industrial RFID application for asset management. The paper by Lopez et al., "Taxonomy, Technology and Applications of Smart Objects" Lopez et al. (2009), discusses the concept of smart objects. The authors propose a classification of smart objects, overview the key enabling technologies, and depict some promising applications of smart objects.

The remaining three articles accepted in this special issue will appear in a separate issue as regular papers due to space constraint. The paper by Li et al., "TMSRFID: Temporal Management of Large-Scale RFID Applications" Li et al. (2009), presents TMS-RFID, a system for temporal management of RFID applications over high-speed RFID data streams. The authors propose a comprehensive model based on the notion of time to live (TTL), for efficient handling of RFID data streams. They also develop a new data structure, namely DLSlist, to solve the problem of unordered event streams. The paper by Hsu et al., "Threshold Jumping and Wrap-Around Scan Techniques toward Efficient Tag Identification in High Density RFID Systems" Hsu et al. (2009), deals with the challenge in identifying high density RFID tags in large-scale RFID systems. The authors propose two enhanced tree-based tag identification techniques, namely Threshold Jumping and Wrap-around Scan to minimize tag identification cost and improve the performance. The paper by Choi and Oh, "Tag-Only Aging-Counter Localization for the R-LIM2 System" Choi and Oh (2009), addresses the localization problem of books in libraries. They present a cost-effective RFID-based Library Management (RF-LIM) system that makes use of inexpressive RFID tags. The RF-LIM system exploits their proposed aging-counter localization algorithm which helps to identify the relative location of books and signals their locations to users. This interesting localization approach can be applied not only to locate books in libraries but also to other interesting applications such as patient tracking and robot positioning.
The papers included in this special issue cover a wide range of topics and present some of the key directions in this vibrant and rapidly expanding area of research and development. We hope that the set of selected papers provides the community with a better understanding of the current directions and areas to focus in future, and inspires your own work.

Acknowledgements We thank all the authors for considering this special issue as an outlet to publish their research results in the area of RFID. We also would like to thank the referees who provided very useful and thoughtful feedback to the authors. Finally, we express our gratitude to the Editors-in-Chief, Professor Rao and Professor Ramesh for their kind support, advice, and encouragements throughout the preparation of this special issue.

\section{References}

Choi, J.-W., \& Oh, D.-I. (2009). Tag-only aging-counter localization for the R-LIM2 system. Information Systems Frontiers, forthcoming.

Hsu, C.-H., Chao, H.-C., \& Park, J. H. (2009). Threshold jumping and wrap-around scan techniques toward efficient tag identification in high density RFID systems. Information Systems Frontiers, forthcoming.

Li, X., Liu, J., Sheng, Q. Z., Zeadally, S., \& Zhong, W. (2009). TMS-RFID: Temporal management of large-scale RFID applications. Information Systems Frontiers, forthcoming.

Lopez, T. S., Ranasinghe, D. C., Patkai, B., \& McFarlane, D. (2009). Taxonomy, technology and applications of smart objects. Information Systems Frontiers Special Issue on RFID, forthcoming.

Meng, S., Chiu, D., Kafeza, E., Liu, W., \& Li, Q.(2009). Automated management of assets based on RFID triggered alarm messages. Information Systems Frontiers Special Issue on RFID, forthcoming.

Mitrokotsa, A., Rieback, M. R., \& Tanenbaum, A. S. (2009). Classifying RFID attacks and defenses. Information Systems Frontiers Special Issue on RFID, forthcoming.

Schwieren, J., \& Vossen, G. (2009). ID-services: An RFID middleware architecture for mobile applications. Information Systems Frontiers Special Issue on RFID, forthcoming.

Sengupta, A., \& Schiller, S. Z. (2009). FlexRFID: A design, development and deployment framework for RFID-based business applications. Information Systems Frontiers Special Issue on RFID, forthcoming.

Sheng, Q. Z., Zeadally, S., Luo, Z., Jung, J.-Y., \& Maamar, Z. (2009). Ubiquitous RFID: Where are we? Information Systems Frontiers Special Issue on RFID, forthcoming.

Ziekow, H., \& Günther, O. (2009). Sharing RFID and complex event data among organizations. Information Systems Frontiers Special Issue on RFID, forthcoming.

Zhou, S., Zhang, Z., Luo, Z., \& Wong, E. C. (2009). A lightweight anti-desynchronization RFID authentication protocol. Information Systems Frontiers Special Issue on RFID, forthcoming.

Zuo, Y. (2009). Secure and private search protocol for RFID systems. Information Systems Frontiers Special Issue on RFID, forthcoming. 
Quan Z. Sheng is currently a lecturer in the School of Computer Science at the University of Adelaide. He holds a $\mathrm{PhD}$ degree in computer science from the University of New South Wales (UNSW) and did his post-doc as a research scientist at CSIRO ICT Centre. His main research interests include Web services, Internet computing, and pervasive computing. $\mathrm{He}$ is the founding chair of the International Workshop on RFID Technology. Dr. Sheng has edited nine books and proceedings and published more than 50 refereed technical papers in journals and conferences including VLDB Journal, IEEE Transactions on Services Computing, IEEE Computer, IEEE Internet Computing, Communications of the ACM, VLDB, ICDE, ICSE, and CAiSE. He is the program co-chair of IEEE SITIS08, publication chair of WISE05, PDCAT07, and publicity co-chair of ICSOC05, WISE07. Dr. Sheng is the recipient of the Microsoft Research Fellowship (2003-2004) and CSC Fellowship (1999-2000). He is a member of the IEEE and the ACM.

Sherali Zeadally received his Bachelors Degree in Computer Science from University of Cambridge, England, and the Doctoral Degree in Computer Science from University of Buckingham, England, in 1996. He is an Associate Professor at the University of the District of Columbia. He currently serves on the Editorial Boards of 12 international journals. He has been serving as a Co-Guest editor for over a dozen special issues of various peer-reviewed scholarly journals. He is a Fellow of the British Computer Society and a Fellow of the Institution of Engineering Technology, UK. His research interests include computer networks including wired and wireless networks), network and system security, mobile computing, ubiquitous computing, RFID, performance evaluation of systems and networks.

Zongwei Luo is a senior researcher of the E-business Technology Institute, the University of Hong Kong, Hong Kong, China. Before that, he was with IBM TJ Watson Research Center in Yorktown Height, NY, USA. He also served as the Affiliate Senior Consultant to ETI Consulting Limited. His research has been supported by various funding sources including China NSF,
HKU seed funding, HK RGC, and HK ITF. His research results have appeared in major international journals and leading conferences. He is the founding Editor-in-Chief of the International Journal of Applied Logistics. He serves as associate editor and editorial advisory board member in many international journals. Dr. Luo's recent interests include applied research and development in the area of Service Science and Computing, innovation management and sustainable development, technology adoption and risk management, and e-business model and practices, especially for logistics and supply chain management.

Jen-Yao Chung received the M.S. and Ph.D. degrees in computer science from the University of Illinois at UrbanaChampaign. He is the senior manager for Industry Technology and Solutions, IBM T. J. Watson Research Center, responsible for identifying and creating emerging solutions with focus on "Green Computing and Business". Before that, he was Chief Technology Officer for IBM Global Electronics Industry. Before that, he was senior manager of the electronic commerce and supply chain department, and program director for the IBM Institute for Advanced Commerce Technology office. Dr. Chung is co-Editor in Chief of the International Journal of Service Oriented Computing and Applications (published by Springer). Dr. Chung is the co-founder and co-chair of the IEEE technical committee on Electronic Commerce. He has served as general chairs and program chairs for many international conferences. He has authored or co-authored over 160 technical papers in published journals or conference proceedings. He is a Fellow of IEEE and a Distinguished Engineer of ACM.

Zakaria Maamar received his Ph.D. in Computer Sciences from Laval University, Canada in 1998. Currently, he is a Professor in the College of Information Technology at Zayed University, Dubai, United Arab Emirates. His research interests lie in the areas of context-aware computing, Web services, and software agents. 